\title{
Prevention of Inorganic Arsenic induced Squamous Cell Carcinoma of Skin in Swiss Albino Mice By Black Tea Through Epigenetic Modulation
}

\section{Archismaan Ghosh}

Chhittaranjan National Cancer Institute

Madhumita Roy

Chhittaranjan National Cancer Institute

Ansuman Lahiri

University of Calcutta

Sutapa Mukherjee

Chhittaranjan National Cancer Institute

Amitava Datta ( $\nabla$ amitava.datta@uwa.edu.au )

University of Western Australia

\section{Research Article}

Keywords: inorganic arsenic, Squamous Cell Carcinoma, methyltransferases, demethylases, acetyltransferases, Black Tea Extract, JARID1B

Posted Date: October 25th, 2021

DOl: https://doi.org/10.21203/rs.3.rs-966459/v1

License: (c) (1) This work is licensed under a Creative Commons Attribution 4.0 International License.

Read Full License 
Prevention of Inorganic Arsenic induced Squamous Cell Carcinoma of Skin in Swiss albino mice by Black Tea through Epigenetic Modulation

Archismaan Ghosh ${ }^{1}$, Madhumita Roy $^{1}$, Ansuman Lahiri ${ }^{2}$, Sutapa Mukherjee ${ }^{1}$, Amitava Datta $^{3 *}$

${ }^{1}$ Dept Environmental Carcinogenesis \& Toxicology

Chhittaranjan National Cancer Institute

37, S P Mukherjee Road

Kolkata 700026, INDIA

${ }^{2}$ Department of Biophysics, Molecular Biology \& Bioinformatics

University of Calcutta

92 APC Road, Kolkata 700009, INDIA

${ }^{3}$ Department of Computer Science and Software Engineering

University of Western Australia

Perth WA 6009, AUSTRALIA

*Department of Computer Science and Software Engineering

University of Western Australia

Perth WA 6009, AUSTRALIA

amitava.datta@uwa.edu.au 


\begin{abstract}
:
Consumption of inorganic Arsenic (iAs) beyond safe levels leads to many diseases including cancers of skin. Carcinogenicity of iAs is mediated through the generation of excessive reactive oxygen species. Previous studies have shown that molecules present in black tea extract (BTE) can ameliorate many deleterious effects of iAs on genetic pathways. This study examines whether BTE can reduce deleterious epigenetic effects due to iAs in Swiss albino mice. We used three groups of mice, a control group, a group that was administered iAs and a group that was administered iAs and BTE. Invasive squamous cell carcinoma (SCC) developed in the iAs group after 330 days, but mice in the iAs+BTE group developed only hyperplasic and dysplastic changes. We report on expression levels of several histone methylation and acetylation marks, as well as those of several histone methylases, demethylases and acetylases. Several aberrant expression levels due to iAs were modulated by BTE. However, the expression level of JARID1B, a histone demethylase and a key marker of SCC was not modulated by BTE, though its demethylation activity was reduced by BTE. in silico studies using docking and molecular dynamical simulation showed that theaflavin compounds present in BTE are excellent inhibitors of JARID1B.
\end{abstract}

Keywords : inorganic arsenic, Squamous Cell Carcinoma, methyltransferases, demethylases, acetyltransferases, Black Tea Extract, JARID1B 


\section{Introduction}

Genetic mutation, epigenetic modulation and environmental factors contribute to carcinogenesis. Chronic exposure to inorganic arsenic, a class I carcinogen declared by IARC ${ }^{1}$, beyond the safe level $(10 \mu \mathrm{g} / \mathrm{L}$, as set by $\mathrm{WHO}^{1}$ ), leads to numerous diseases including cancers of the skin, bladder, kidney and liver. Arsenic mediates its carcinogenicity by excessive generation of reactive oxygen species (ROS) ${ }^{2}$. Metabolism of inorganic arsenic (iAs) utilises S-adenosyl methionine (SAM), whose depletion occurs due to chronic exposure to iAs, altering global methylation pattern of DNA and gene expression ${ }^{3}$. iAs is also known to induce different types of epigenetic modulations, and the present study focuses on post-translational histone modifications (PTM) including histone methylation, demethylation and acetylation. The main contributions of this study are two-fold. The carcinogenic effect of iAs on squamous cell carcinoma (SCC) due to PTM and the alleviation of these effects by black tea extract (BTE) have been studied extensively in vivo in the mouse model. The effects of iAs on several of these methylases and demethylases have not been reported before to the best of our knowledge. This paper also establishes the potential of theaflavin compounds (present in BTE) as inhibitors of JARID1B, a di- and tridemethylase of $\mathrm{H} 3 \mathrm{~K} 4$ through extensive in silico studies using protein-ligand docking and molecular dynamical simulations.

Antioxidants mitigate the carcinogenicity of iAs due to ROS, as generation of ROS drives epigenetic alterations ${ }^{4}$. The genetic and epigenetic modulation by phytochemicals have been reviewed extensively in the context of carcinogenesis $^{5}$. Tea contains polyphenols and is a good source of antioxidants ${ }^{6}$. Major constituents of black tea are theaflavins (TF) and thearubigins (TR). TF, a seven-member benztropolone rings is formed due to cooxidation of pairs of epimerized catechins. This molecule is capable of chelating transition metals, thereby countering free radical generation. Resonance in the benztropolone moieties quench oxidative species by donating an electron ${ }^{7}$. Of the three main derivatives of TF (theaflavin-3-gallate, theaflavin-3'-gallate, and theaflavin-3-3'digallate), theaflavin-3-gallate can inhibit the activity of DNA methyltransferase-3a(DNMT3a) by directly binding. ${ }^{8}$ Theaflavin-3-3'-digallate can directly interact with model histone proteins, double stranded and quadruplex DNA strands. Therefore, action of TF is due to its translocation to the nucleus and interaction with different nuclear proteins and DNA ${ }^{8}$.

Studies on the influence of iAs on these histone proteins, particularly for SCC of skin in mice model have not been reported much in literature. This study extensively examines the role iAs plays in epigenetic regulation of SCC. Furthermore, this in vivo study indicates that BTE may have inhibiting effects on the histone demethylase JARID1B indirectly, not by reducing its expression level, rather by inhibiting its demethylating activities. As JARID1B is an important marker for several skin cancers including SCC, we conducted extensive in silico studies using protein-ligand docking and molecular dynamical simulations using theaflavin compounds and JARID1B. As JARID1B is a therapeutic target for several cancers, finding small molecule inhibitors for JARID1B is an active research area. This study contributes significantly in that direction. Present study aims to investigate the influence of chronic exposure to iAs on the expression of PTM of several histones and their histone methyl transferases (HMT), histone demethylases (HDM) and histone acetyl transferases (HAT) in squamous cell carcinoma and their modulation by BTE. 


\section{Methods}

\subsection{Treatment protocol}

4-5 weeks old, male, Swiss albino mice (Mus musculus) were taken from the animal house of Chittaranjan National Cancer Institute (CNCI), with approval from the Institutional Animal Ethics Committee (IAEC 1774/MR- 3/2017/9). Protocols have been followed according to the standard guidelines laid down by the IAEC, certified by CPCSEA (Committee for the Purpose of Control and Supervision of Experiments on Animals), New Delhi. All the mice were handled and experiments done following the ARRIVE protocols.

Mice were fed with synthetic pellets and maintained in alternate 12 hours of light and darkness at a temperature of $22 \pm 2^{\circ} \mathrm{C}$. All the mice were kept at the same level of the rack, to maintain identical housing condition. Mice were euthanized by an overdose of thiopentone sodium $(100 \mathrm{mg} / \mathrm{kg}$ body $\mathrm{wt})$.

The animals were divided into three groups, each group consisting of 25 mice, kept in separate cages, each cage harbouring 5 mice. Mice were treated with iAs and BTE and tissues and blood were collected at different time intervals. Three groups are: I. Control group, was neither administered iAs, nor BTE. Group II mice were exposed to iAs, whereas, group III mice were exposed to iAs and at the same time were fed with BTE. iAs dose was 500 $\mu \mathrm{g} / \mathrm{l}$ (arsenite) dissolved in water; administered orally as only source of water, plus painted on the shaved hind part, once daily. Group III were treated with iAs, as in group II, along with BTE $(0.33 \mathrm{mg} / \mathrm{gm}$ body weight, lyophilised extract dissolved in water), thrice daily by oral gavage, at an interval of 8 hours.

Developmental stages of Squamous cell carcinoma (SCC) of the skin of mice were confirmed by histological analysis.

\subsection{Preparation of black tea extract}

$2.5 \%$ infusion of Black tea was prepared in boiling water and cooled. It was lyophilized in a SCANVAC lyophilizer. The lyophilized product was weighed, reconstituted in water and administered to the mice by gavage (at an interval of $8 \mathrm{hrs}$, cumulated dose being $0.33 \mathrm{mg} / \mathrm{gm}$ body weight per day) ${ }^{5}$. The polyphenolic content of tea was determined by HPLC.

\subsection{Quantification of Tea polyphenols using HPLC}

HPLC was carried out using a $515 \mathrm{lb}$ dual pump with a control (Waters, USA), a Rheodyne injector, a RP C-18 column by Nova-Pak which was attached to the guard column regulated at $30^{\circ} \mathrm{C}$ and a $996 \mathrm{PDA}$ detector (Waters, USA) adjusted to $278 \mathrm{~nm}$. Catechins were separated using gradient of 5-25\% acetonitrile in $0.025 \mathrm{M} \mathrm{KH}_{2} \mathrm{PO}_{4}, \mathrm{pH}$ 2.4. Isocratic mode was used to separate theaflavin, where the mobile phase consisted of acetic acid: acetone: water in the ratio of 1:60:39 and detected at $365 \mathrm{~nm}$. Integration and calibration of mixture containing catechins and theaflavins were done using the software Millenium 32. The quantification of the content of catechins and theaflavins was done by plotting against the standard.

\subsection{Confirmation of skin cancer by histology}

The skin tissues collected after sacrificing the mice, were fixed in 10\% Neutral Buffered Formalin (NBF). They were then treated with increasing grades of alcohol $(50 \%, 70 \%, 90 \%$ and 100\%) and xylene to remove water and fat content respectively. The tissues were then embedded in paraffin and cut into $4 \mu \mathrm{m}$ thick sections using a microtome (Leica). These tissue sections were stretched on glass slides, stained with haematoxylin and eosin, 
mounted with DPX and observed under light microscope (Zeiss). The nucleus appeared blue due to haematoxylin staining on a pink background of the cytoplasm, due to counter-staining by eosin. The development of squamous cell carcinoma in the mice skin was confirmed by histology analysis.

\subsection{Histone extraction from animal tissues}

The isolated skin tissues were homogenised in Dounce homogeniser in Triton-X Extraction Buffer (0.5\% Triton$\mathrm{X}, 2 \mathrm{mM}$ PMSF, $0.02 \% \mathrm{NaN}_{3}$ ). The mixture was centrifuged at $10,000 \mathrm{rpm}$ for 1 minute at $4^{\circ} \mathrm{C}$. The pellet was suspended in extraction buffer $(0.5 \mathrm{~N} \mathrm{HCl}$ and $10 \%$ glycerol $)$. After centrifugation at 12,000 rpm for 5 minutes at $4^{\circ} \mathrm{C}$, supernatant was collected and kept overnight with $600 \mu \mathrm{l}$ of acetone at $-20^{\circ} \mathrm{C}$. Finally, the pellet after centrifugation at $12,000 \mathrm{rpm}$ was suspended in deionised water and stored at $-80^{\circ} \mathrm{C}$ after protein estimation using Bradford's method.

\subsection{H3K4 global methylation detection:}

Global histone methylation was assessed colorimetrically from the isolated histone extracts. EpiQuikTM Global Histone H3K4 Methylation Assay Kit (P-3017-96, Epigentek) was used. The absorbance OD was measured using a microplate reader Tecan infinite-200 PRO, at $450 \mathrm{~nm}$ and the methylation (\%) was calculated. [Methylation $\%=\{\mathrm{OD}$ (Sample- blank)/OD (untreated control- blank) $\} \times 100]$.

\subsection{Western Blot analysis:}

Western blot analysis was carried out using the isolated histone proteins, following laboratory protocol ${ }^{2}$. The western blot bands were scanned and quantified by using IMAGE MASTER Software (Amersham Pharmacia biosciences, USA).

\subsection{Immunohistochemistry analysis:}

Immunohistochemistry was performed on formalin fixed, paraffin embedded tissues. Tissues were incubated overnight with primary antibody at $4^{\circ} \mathrm{C}$, washed with PBS and stained with secondary antibody HRP-conjugated for half an hour at room temperature. It was then stained with diaminobenzidine (DAB), mounted in DPX and observed under microscope. The expressions of various proteins in IHC were analyzed ${ }^{8}$. Minimum of 15 fields at 400x were visualised under a light microscope. The expressions of various proteins in IHC were analyzed using the scoring system as formulated by Allred et al $1998^{9}$. The scoring system classifies the percentage of stained cells into 6 proportion scores (PS) and the intensity of staining into 4 intensity scores (IS). The final Allred score is calculated as : AS = PS + IS. The Allred score can be categorized into 9 categories (0-8), the highest score being 8 (PSmax + ISmax). Category of proportion scores (PS) of 0, 1, 2, 3, 4, 5 correspond to the percentage of stained cells $0,<1,1-10,11-33,34-66$ and $\geq 67$ respectively. The intensity score (IS) was divided into four categories depending on the intensity of the staining. IS of $0,1,2,3$ correspond to no stain, weak stain, intermediate stain and strong stain respectively. Each section was scored 5 times and the highest AS was reported.

\subsection{Docking studies:}

The crystal structure of JARID1B (KDM5B) (PDB ID: 5FUN) was obtained from protein data bank (https://www.rcsb.org). This structure was used for studying small molecule inhibitors for the JmjC domain of JARID1B $^{10}$. The chemical structures of theaflavin (PubChem CID: 135403798) and theaflavin-3,3'-digallate 
(PubChem CID: 21146795) were downloaded from PubChem ((https://pubchem.ncbi.nlm.nih.gov/). Protein and ligand preparations were done using Python Molecular Viewer (pmv), part of MGLTools from Scripps Institute ${ }^{11}$. The PHD1-ARID domains were deleted from JARID1B structure (PDB ID: 5FUN) and it was considered suitable for our study as the focus is on the JmjC domain. Johansson et al. ${ }^{9}$ used several supplementary ligands in their study which were removed from the PDB file for molecular dynamical simulations. These supplementary ligands are EDO (1,2 ethanediol), EPE (4-(2-hydroxyethyl)-1-piperazine ethanesulfonic acid), GZA (2-[(1-benzyl-1Hpyrazol-4-yl)oxy]pyrido[3,4-d]pyrimidin-4(3H)-one), metals ( $\mathrm{Mn}, \mathrm{Zn}, \mathrm{Na}$ ) and phosphate ion. The removal of these supplementary ligands had no effect on the protein structure, which was verified by superimposing the two structures. The protein was prepared by deleting water molecules, adding polar hydrogen and Gasteiger charges, and saved in the pdbqt format. The ligand was prepared by adding Gasteiger charges and saved in the pdbqt format. Docking was done using Webina ${ }^{12}$, a web-based docking tool that runs in a browser using AutoDock Vina $^{13}$. The centre $(x=88, y=66, z=11.6)$ and size $(x=30, y=27, z=32)$ of the docking box were chosen to cover the JmjC domain of JARID1B. The top-9 docking poses for both theaflavin and theaflavin-3,3'-digallate were analyzed for polar interactions using PyMol. The docking poses were extracted from the output using vina_split, a tool in the AutoDock Vina software.

\subsection{Molecular Dynamical Simulation:}

Preparations of the protein-ligand systems for molecular dynamical simulation were done using Gromacs in-built tools $^{14}$ version 2020.1-Ubuntu-2020.1-1 on a 64-bit, quad core Ubuntu system. The Bioexcel Building Block library [https://mmb.irbbarcelona.org/biobb/availability/tutorials/md_setup] workflow was used for preparation of the protein-ligand system, using python 3.7 and Jupyter Notebook. The Amber ff99sb forcefield was used, as this forcefield is considered to be appropriate for protein-ligand simulations. Short (10 picosecond) molecular dynamical simulations were done at the preparation stage to check the setup parameters.

The Zeus supercomputer in Pawsey Supercomputing Facility in Perth, Western Australia was used for the Gromacs production runs (Intel Xeon E5-2690V3 "Haswell" processors, 12-cores, 2.6 Ghz). 24 MPI processes and 4 OpenMP threads in each MPI process were found to provide optimal performance. Each production simulation was run for 24 hours, providing approximately 37 nano seconds of simulation time.

Molecular mechanics Poisson-Boltzmann surface area (MM/PBSA), a widely used method to calculate the binding free energy between protein and ligands was used. Although it is not as accurate as alchemical free energy methods, it is considered to be more accurate than scoring functions used in docking and substantially much faster than the alchemical methods. MM/PBSA analysis was done using the g_mmpbsa tool ${ }^{15}$. The docking movies were created using Gromacs trajectory files.

\subsection{Statistical analysis:}

Statistical analysis was done using SPSS 10.0 (one-way analysis of variance followed by Dunett's t-test). Significance level of $\mathrm{p}<0.001$ was accepted. Comparison between the groups was done using unpaired t-test. 


\section{Results}

\subsection{Polyphenol content of BTE:}

The polyphenolic contents in BTE were quantitated. Different constituents of Catechins, EGCG [(-)epigallocatechin gallate, EGC [(-)- epigallocatechin] and ECG [(-)- epicatechin gallate] were separated using gradient mode; whereas TF [theaflavin] was separated in isocratic mode. Concentrations $(\mathrm{mg} / \mathrm{ml})$ of the polyphenolic content of BTE used for the study are 0.24, 0.43, 0.30 and 0.97 for EGC, EGCG, ECG and TF respectively.

\subsection{Development of carcinoma:}

Development of SCC of skin by chronic iAs exposure is depicted in Figure 1. Mice receiving co-treatment of BTE with iAs developed papillomatous lesions, with hyperplasic or mild dysplastic changes, as evident from histology. No cytological atypia or massive infiltration of the dermal layer was observed indicating no development of invasive SCC even at 330 days of treatment.

Minor lesions started appearing in the skin tissues of the iAs treated mice after 90 days of treatment. These lesions further aggravated and developed into major invasive gaping wounds at 330 days. Histopathological analysis of the iAs treated mice revealed the loss of entire epidermal structure and prominent infiltration of the deeply stained, aggressive nuclei, covering more than $2 / 3$ rd proportion of the underlying dermis, confirming invasive squamous cell carcinoma. 


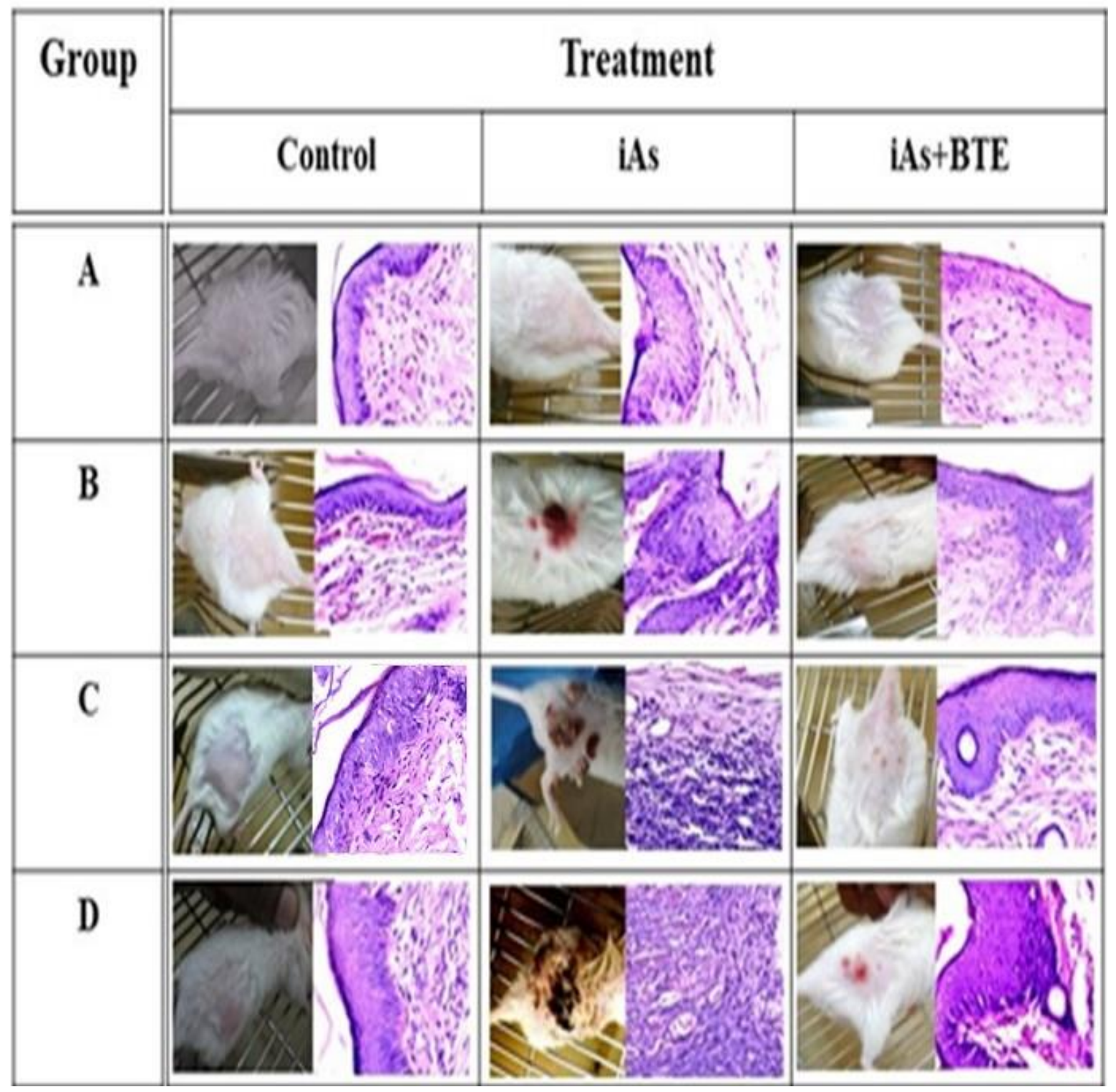

Figure 1. Development of skin carcinoma. Swiss albino mice were chronically exposed to iAs (500 $\mu \mathrm{g} / \mathrm{l})$. Three groups of mice were sacrificed at (A) 30, (B) 180, (C) 270 and (D) 330 days. Histological analysis revealed (A) no visible changes in control; iAs+BTE treated mice; slight thickening of the epidermal layer was observed in iAs group. (B) no changes in control tissue; in iAs group prominent hyperplasic and dysplastic changes were observed in the epidermal layer; mild hyperplasia was observed in iAs+BTE group. (C) no prominent changes were observed in the epidermal layer; in iAs formation of in situ carcinoma was observed with regions of the tissue showing loss of epidermal architecture and presence of deeply stained nuclei; whereas in iAs+BTE group prominent hyperplasic changes were observed with presence of frequent keratin pearls. (D) mild hyperplasic changes were observed, due to aging; in iAs treated group more than two-third of the tissue sections show complete loss of the epidermal architecture, filled with deeply stained nuclei indicating invasive carcinoma; in iAs+BTE thickening of the epithelium, presence of keratin pearls along with other dysplastic changes were clearly visible in the tissue.

\subsection{Global methylation status of H3K4:}

Global methylation status of H3K4 (Figure 2) indicates hypomethylation due to iAs, which can be significantly prevented $(\mathrm{p}<0.0001)$ by BTE. Colorimetric estimation of the global methylation status of H3K4 loci revealed a trend of demethylation with chronic iAs exposure. The methylation percentage of H3K4 was significantly low $(\mathrm{p}<0.0001)$ in iAs treated group with respect to the control group. The BTE administered mice showed significantly higher $(\mathrm{p}<0.0001)$ methylation percentage at 330 days of treatment with respect to the iAs group, indicating inhibition of loss of global methylation at H3K4 loci by BTE. 


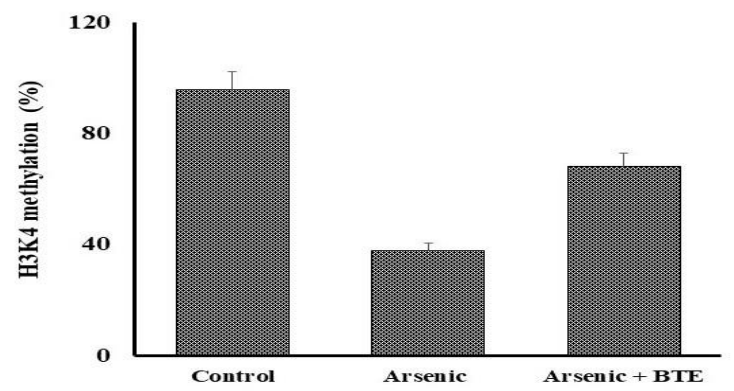

Figure 2. Global methylation at H3K4 loci. Global methylation has been depleted to 0.4-fold by exposure to iAs for 330 days. Reduced methylation at H3K4 loci has been increased by BTE administration. Values are average of three independent experiments \pm SD. Reduction in global H3K4 methylation is significant with respect to control at $p<0.0001$; enhancement of the same by BTE is significant at $\mathrm{p}<0.0001$.

\subsection{Expression of epigenetic histone marks and their modifiers (methyltransferases, demethylases and acetyltransferases):}

Western blot analysis reveals alteration in expression of histone modifiers by chronic iAs exposure for 330 days and their modulation by BTE (Figure 3). Significant increase in the expression of H3K4me1, the methyl transferase MLL3, JARID1B, H3K27me3, EZH2 methyltransferase along with JARID2 upon iAs exposure for 330 days was observed. Intervention with BTE in the group of mice exposed to iAs, significantly brought down the elevated expression levels of these proteins due to iAs, though the extent of down-regulation varied. BTE downregulated their over-expression significantly, except for JARID1B, which is not influenced by BTE. Corresponding significance levels are given in Table 1. Contrastingly, chronic exposure to iAs significantly reduced the expression levels of H3K4me3, MLL1, KDM6A, H4K16ac and its acetyltransferase MYST1. Intervention with BTE upregulated the expression of these histone proteins and their modifiers. Expression of demethylase LSD1 however is not influenced either by iAs or iAs+BTE. Corresponding band intensities have been given in Figure 4. 


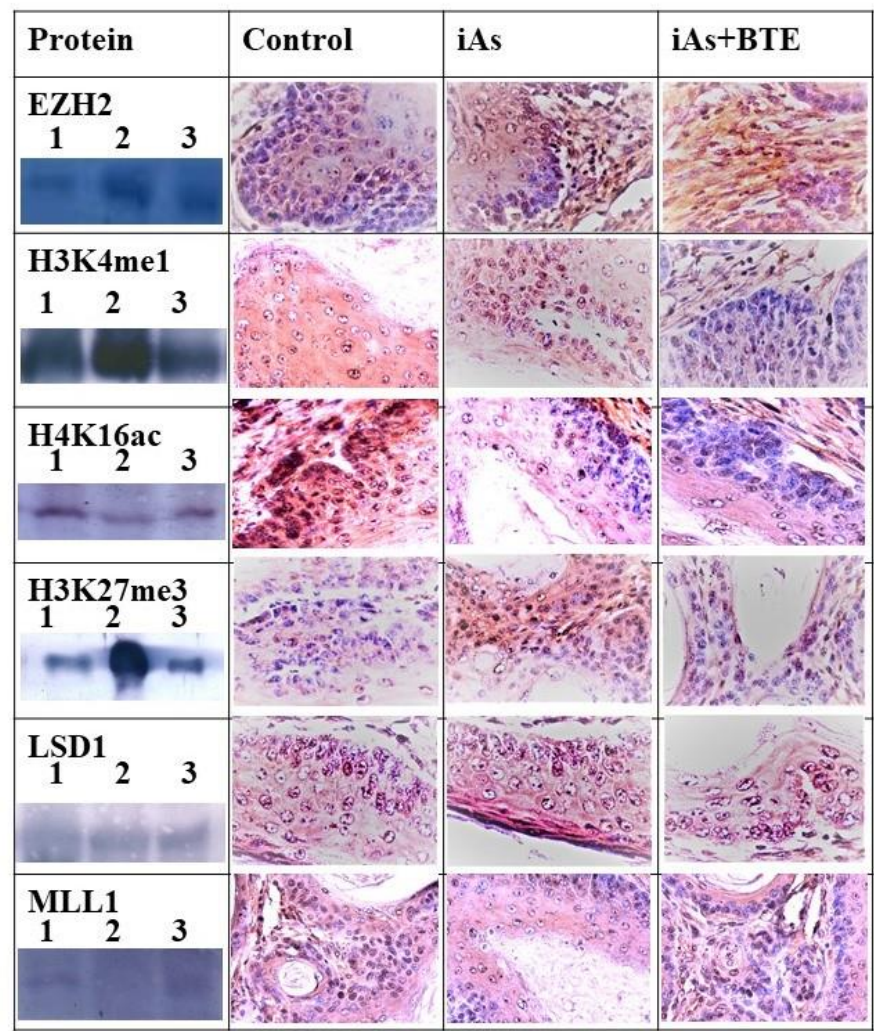

IHC

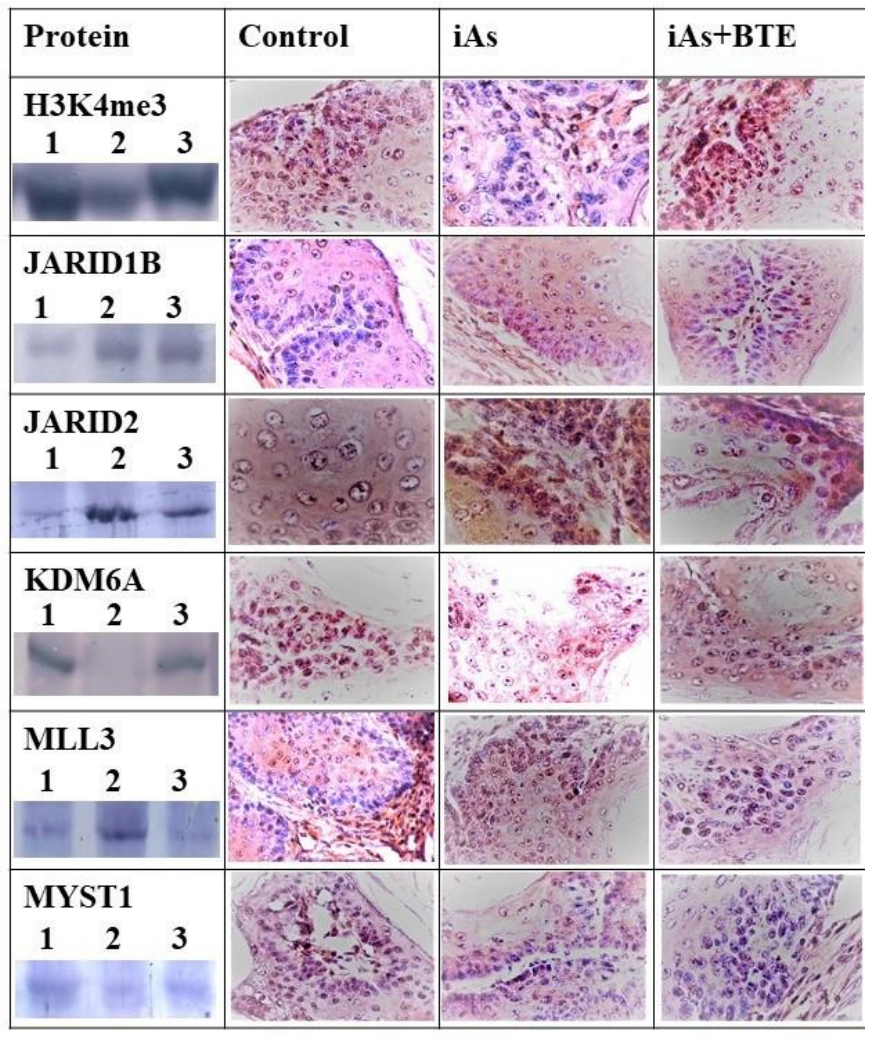

IHC

Lane 1- Control; Lane 2- iAs; Lane 3- iAs+BTE

Figure 3. Influence of iAs on the expression of epigenetic histone marks and their modifiers (methyltransferases, demethylases and acetyltransferases). Western blot bands and corresponding IHC results from skin tissue of mice after 330 days of exposure using corresponding antibodies have been depicted. In Western blot bands, Lane 1, 2, 3 represent control, iAs and iAs+BTE treated mice respectively. Bands have been cropped from full length gels, which have been provided in the Supplementary Info File. In IHC, formalin fixed, paraffin embedded section of skin tissues after incubation with primary antibody were stained with DAB. Images were captured at $400 \mathrm{x}$.

\section{图 Control BiAs 口iAs+BTE}

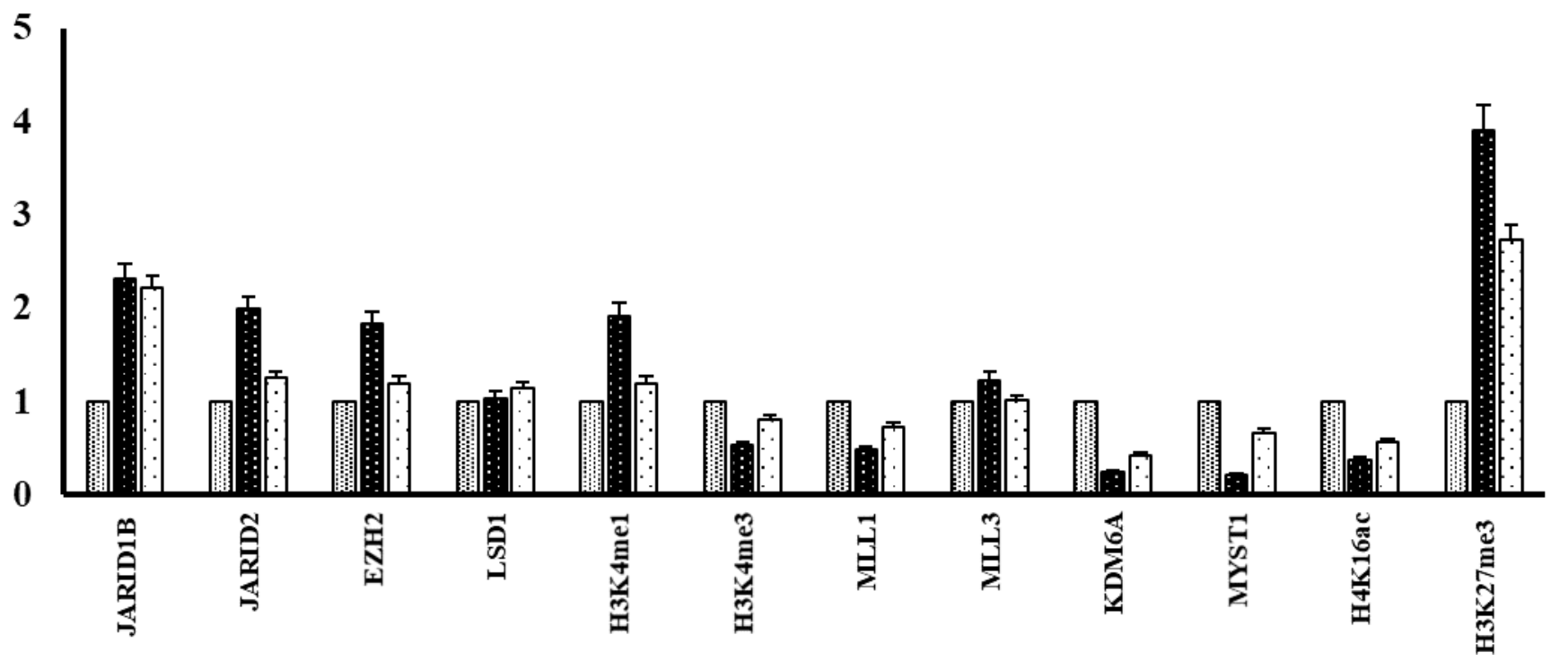

Figure 4. Modulation of various histone marks by iAs during skin carcinogenesis, as modulated by BTE. Band intensities (fold change) as obtained from Western blot results (from Figure 3) have been represented in a bar diagram. Values are the mean band intensities of three independent experiments \pm SD. 
Table 1. Significance of histone mark modulation by iAs and their alteration by BTE

\begin{tabular}{|l|c|c|c|c|}
\hline \multirow{2}{*}{ Proteins } & \multicolumn{4}{|c|}{ Significance (p values) } \\
\cline { 2 - 5 } & \multicolumn{3}{|c|}{ Western blot } & \multicolumn{2}{c|}{ Allred score } \\
\cline { 2 - 5 } & $\begin{array}{l}\text { Expression level } \\
\text { after iAs exposure, } \\
\text { wrt control }\end{array}$ & $\begin{array}{l}\text { Expression level } \\
\text { after intervention } \\
\text { with BTE, with } \\
\text { respect to iAs } \\
\text { exposure }\end{array}$ & $\begin{array}{l}\text { Expression level } \\
\text { after iAs exposure, } \\
\text { wrt control }\end{array}$ & $\begin{array}{l}\text { Expression level } \\
\text { after intervention } \\
\text { with BTE, with } \\
\text { respect to iAs } \\
\text { exposure }\end{array}$ \\
\hline PLU1/JARID1B & $\uparrow \mathrm{p}<0.0001$ & $\mathrm{p}=0.6787$ & $\uparrow \mathrm{p}<0.0001$ & $\mathrm{p}=0.1442$ \\
\hline JARID2 & $\uparrow \mathrm{p}<0.0001$ & $\downarrow \mathrm{p}<0.005$ & $\uparrow \mathrm{p}<0.0001$ & $\downarrow \mathrm{p}<0.005$ \\
\hline EZH2 & $\uparrow \mathrm{p}<0.0001$ & $\downarrow \mathrm{p}<0.005$ & $\uparrow \mathrm{p}<0.0001$ & $\downarrow \mathrm{p}<0.0001$ \\
\hline LSD1 & $\mathrm{p}=0.4199$ & $\mathrm{p}=0.3000$ & $\mathrm{p}=1.000$ & $\downarrow \mathrm{p}=0.0847$ \\
\hline H3K4me1 & $\uparrow \mathrm{p}<0.005$ & $\downarrow \mathrm{p}<0.005$ & $\uparrow \mathrm{p}<0.0001$ & $\downarrow \mathrm{p}<0.005$ \\
\hline H3K4me3 & $\downarrow \mathrm{p}<0.0001$ & $\uparrow \mathrm{p}<0.005$ & $\downarrow \mathrm{p}<0.0001$ & $\uparrow \mathrm{p}<0.0001$ \\
\hline MLL1 & $\downarrow \mathrm{p}<0.0001$ & $\uparrow \mathrm{p}<0.005$ & $\downarrow \mathrm{p}<0.0001$ & $\uparrow \mathrm{p}<0.005$ \\
\hline MLL3 & $\uparrow \mathrm{p}<0.0001$ & $\downarrow \mathrm{p}<0.005$ & $\uparrow \mathrm{p}<0.005$ & $\downarrow \mathrm{p}<0.005$ \\
\hline KDM6A & $\downarrow \mathrm{p}<0.0001$ & $\uparrow \mathrm{p}<0.005$ & $\downarrow \mathrm{p}<0.0001$ & $\uparrow \mathrm{p}<0.005$ \\
\hline MYST1 & $\downarrow \mathrm{p}<0.0001$ & $\uparrow \mathrm{p}<0.005$ & $\downarrow \mathrm{p}<0.0001$ & $\uparrow \mathrm{p}<0.0001$ \\
\hline H4K16ac & $\downarrow \mathrm{p}<0.0001$ & $\uparrow \mathrm{p}<0.005$ & $\downarrow \mathrm{p}<0.0001$ & $\uparrow \mathrm{p}<0.005$ \\
\hline H3K27me3 & $\uparrow \mathrm{p}<0.0001$ & $\downarrow \mathrm{p}<0.0001$ & $\uparrow \mathrm{p}<0.0001$ & $\downarrow \mathrm{p}<0.0001$ \\
\hline
\end{tabular}

\subsection{IHC evaluation:}

Immunohistochemistry of different histone marks and their modifiers have been depicted in Figure 3. Results are in agreement with western blot analysis. Semiquantitative assessment of immunostaining results was done using the Allred scoring (AS) system, and the final score for each assessed protein has been shown in Table 2. Allred scoring (AS) for JARID1B showed a significant increase in the iAs treated mice, however, no changes were observed upon BTE administration. H3K4me1, MLL3, JARID1B, EZH2, JARID2, H3K27me3 showed a higher score with respect to those of the control group. The Allred score was found to be diminished in the group of mice with iAs and BTE simultaneously. Lowering of AS in H3K4me3, MLL1, KDM6A, MYST1 and H4K16ac was observed in iAs exposed mice for 330 days. A significant rise in AS score was observed in the group of mice exposed to iAs+BTE regularly. Score for LSD1, a H3K4me2 demethylase remained unchanged by iAs, but a slight reduction in score was observed in iAs+BTE group. But, this reduction in LSD1 level was not significant. 
Table 2. Allred Score for IHC of epigenetic markers

\begin{tabular}{|l|l|l|l|}
\hline Proteins & Control & Arsenic (iAs) & iAs + BTE \\
\hline PLU1/JARID1B & $4 \pm 0.63$ & $6 \pm 0.63^{*}$ & $6 \pm 0.91$ \\
\hline JARID2 & $5 \pm 0.45$ & $7 \pm 0.31^{*}$ & $6 \pm 0.45^{\mathrm{b}}$ \\
\hline EZH2 & $4 \pm 0.44$ & $8 \pm 0.21^{*}$ & $6 \pm 0.45^{\mathrm{a}}$ \\
\hline LSD1 & $6 \pm 0.45$ & $6 \pm 0.55$ & $5 \pm 0.62$ \\
\hline H3K4me1 & $5 \pm 0.45$ & $7 \pm 0.38^{*}$ & $6 \pm 0.32^{\mathrm{b}}$ \\
\hline H3K4me3 & $7 \pm 0.38$ & $4 \pm 0.45^{*}$ & $6 \pm 0.45^{\mathrm{a}}$ \\
\hline MLL1 & $7 \pm 0.45$ & $5 \pm 0.50^{*}$ & $6 \pm 0.32^{\mathrm{b}}$ \\
\hline MLL3 & $6 \pm 0.45$ & $7 \pm 0.45^{* *}$ & $6 \pm 0.38^{\mathrm{b}}$ \\
\hline KDM6A & $7 \pm 0.45$ & $5 \pm 0.50^{*}$ & $6 \pm 0.21^{\mathrm{b}}$ \\
\hline MYST1 & $7 \pm 0.45$ & $4 \pm 0.50^{*}$ & $6 \pm 0.50^{\mathrm{a}}$ \\
\hline H4K16ac & $7 \pm 0.45$ & $4 \pm 0.50^{*}$ & $5 \pm 0.32^{\mathrm{b}}$ \\
\hline H3K27me3 & $4 \pm 0.54$ & $8 \pm 0.21^{*}$ & $6 \pm 0.45^{\mathrm{a}}$ \\
\hline
\end{tabular}

Significant at ${ }^{*} \mathrm{p}<0.0001 ;{ }^{* *} \mathrm{p}<0.005$, with respect to the control group. Significant at ${ }^{\mathrm{a}} \mathrm{p}<0.0001 ;{ }^{\mathrm{b}} \mathrm{p}<0.005$, with respect to iAs group.

\subsection{In silico docking studies:}

Initial docking of JARID1B and theaflavin was done in the presence of the supplementary ligands ${ }^{9}$ with high docking affinity. $\mathrm{Fe}$ (II) ion was replaced by $\mathrm{Mn}$ (II) ion by Johansson et al. ${ }^{9}$, and several $\mathrm{C}$ and $\mathrm{H}$ atoms of theaflavin were docked less than $2 \AA$ from the Mn(II) ion. The structure of JARID1B (PDB id: 5FUN) downloaded from PDB with additional ligands and the corresponding cleaned structure of the same are shown in Figure 5. Both theaflavin and theaflavin-3,3'-digallate were docked after removing the supplementary ligands (Figure 5), with high affinities $(-9.7 \mathrm{kCal} / \mathrm{mole}$ and $-11.5 \mathrm{kCal} / \mathrm{mole}$ respectively). Two top poses of both are shown in Figure 5. Analysis using PyMol showed at least one hydrogen bond and several medium range $(<10 \AA)$ polar interactions. Two beta sheets each from either side of the JmjC domain were within $5 \AA$ distance from the docked ligands (theaflavin and theaflavin-3,3'-digallate). 


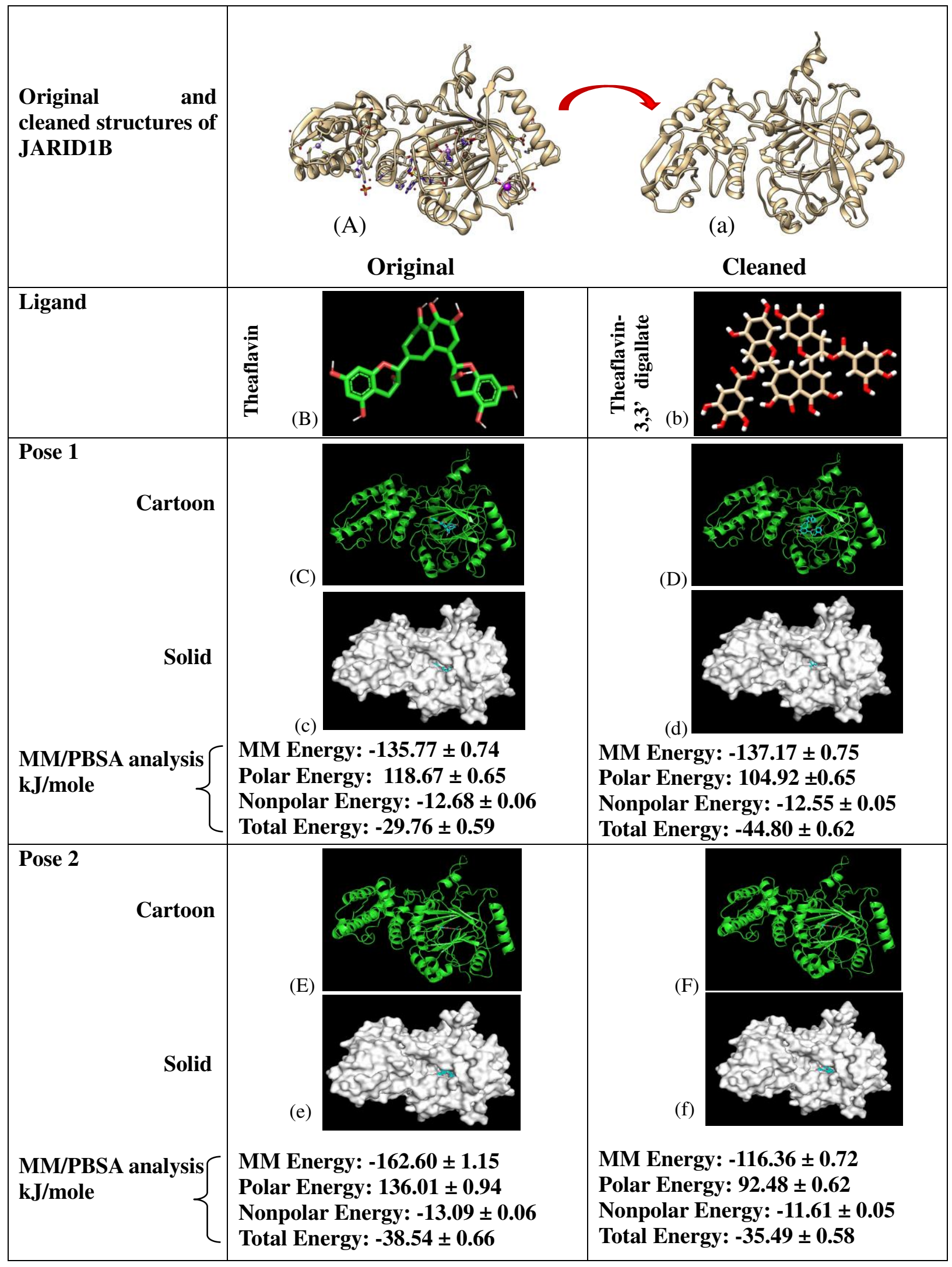

Figure 5. Docking of theaflavin and theaflavin-3,3'-digallate with JARID1B. (A) The structure of JARID1B (PDB id: 5FUN) downloaded from PDB with additional ligands; (a) The cleaned structure of JARID1B. (B) Chemical structure of theaflavin; (b) Chemical structure of theaflavin-3,3'-digallate; (C) and (E) are respectively the top two docking poses (pose 1, pose 2) of theaflavin with JARID1B (cartoon rendering); (c) and (e) are respectively the top two docking poses (pose 1, pose 2) of theaflavin with JARID1B (solid rendering); (D) and (F) are respectively the top two docking poses (pose 1, pose 2) of theaflavin-3,3'-digallate with JARID1B (cartoon rendering); (d) and (f) are respectively the top two docking poses (pose 1, pose 2) of theaflavin-3,3'-digallate with JARID1B (solid rendering). The ligands are shown in cyan colour. 


\subsection{Molecular dynamical simulation:}

MM/PBSA calculations were done on the trajectories of the systems between 30-31 ns from the production run. The time evolution of the RMSDs shows the systems to be fairly stabilized during this period. The lower the calculated binding energy, the stronger the ligand is expected to bind to the protein. From our calculations (Figure 5) it appears that the binding affinity of theaflavin-3,3'-digallate for the JmjC domain of JARID1B is higher than theaflavin.

Time evolution and variation in different energy components as observed from molecular dynamical simulations of JARID1B with theaflavin-3,3'-digallate for the top docking pose are shown in Figure 6. We chose to include the results for the top docking pose of theaflavin-3,3'-digallate due to its higher affinity for docking with JARID1B. The protein-ligand trajectories for the top poses of theaflavin and theaflavin-3,3'-digallate are shown in videos: https://bit.ly/3oObA4s for theaflavin and https://bit.ly/2YzLSWK for theaflavin-3,3'-digallate. 


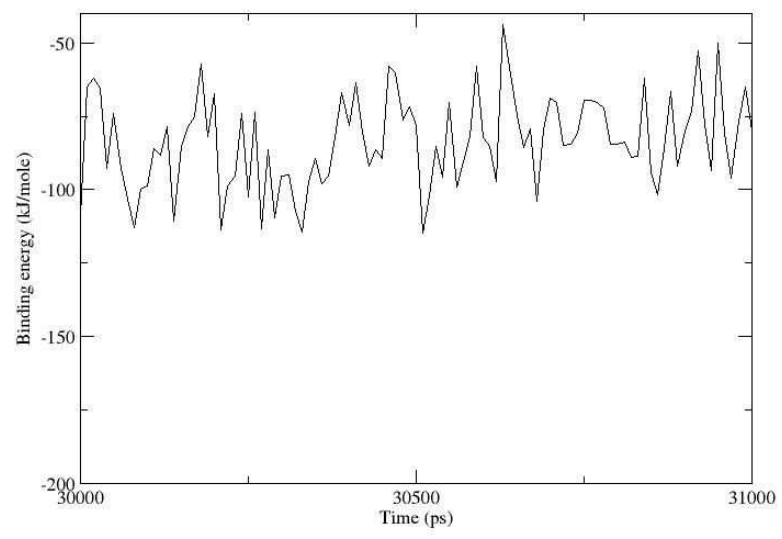

(a)

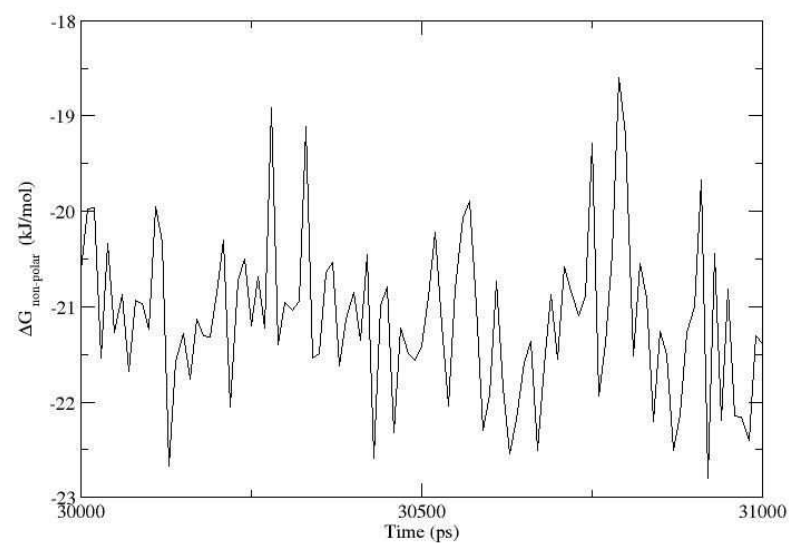

(c)

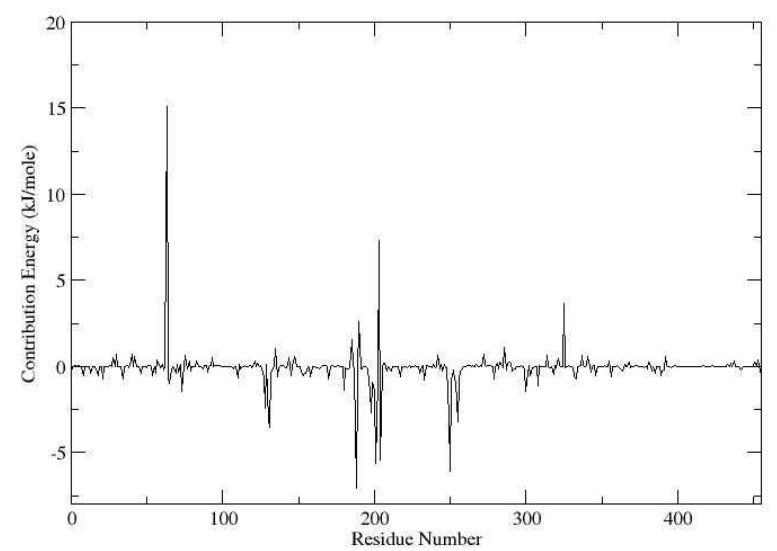

(e)

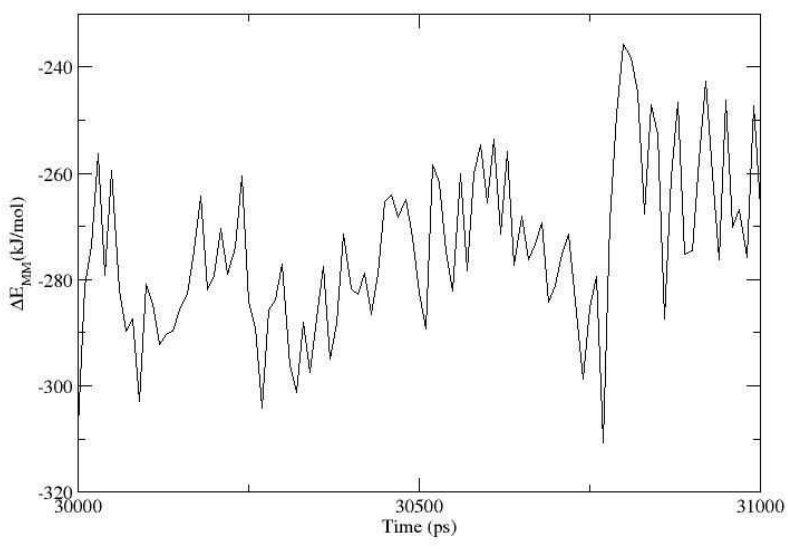

(b)

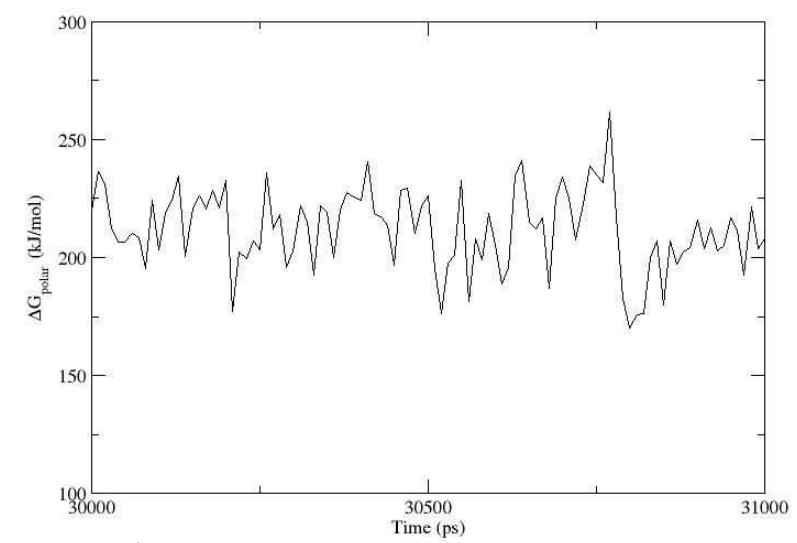

(d)

Hydrogen Bonds

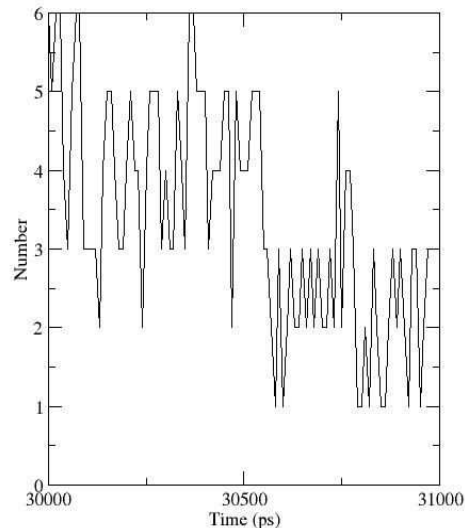

(f)

Figure 6. Analysis of different energy values for the binding of the top pose of theaflavin-3,3'-digallate with JARID1B between 30-31 ns of Gromacs simulation. (a) total binding energy- low total binding energy indicates stable docking; (b) MM energy; (c) non-polar binding energy; (d) polar binding energy; (e) per residue binding energy; (f) hydrogen bonds - the graph shows up to 6 hydrogen bonds, and at least 1 hydrogen bond during the simulation period between 30-31 ns. 


\section{Discussion}

Invasive SCC of skin was observed at 330 days of iAs treatment. With BTE intervention, only hyperplasic and dysplastic changes were observed, where epidermal architecture of the tissue remained mostly intact with the absence of major invasive structures within the dermal layer. PTMs of histones are prognostic cancer markers ${ }^{16}$ and our study focuses on the PTMs of three histone loci, namely H4K16, H3K27 and H3K4, as well as several histone methylases, demethylases and acetyltransferases.

H4K16ac and its acetyltransferase MYST1, have been found to be associated with the promoters of actively transcribed genes. Silencing of MYST1 by iAs reduces H4K16ac, which is involved in DNA damage response ${ }^{17}$. In our study, development of invasive SCC due to iAs, may be partly due to loss of H4K16ac and MYST1, which is in line with other reports ${ }^{18}$. Prevention of invasive SCC by BTE may be partially attributed to the upregulation of H4K16ac and MYST1. Loss of H4K16ac and MYST1 have also been reported in numerous cancers as well as upon exposure to chronic iAs, thereby silencing several genes ${ }^{19,20}$.

$\mathrm{H} 3 \mathrm{~K} 27 \mathrm{me} 3$ is involved in transcriptional silencing ${ }^{21}$ and upregulated in several cancers. H3K27me3, a prognostic marker in various cancers, exhibits negative correlation with patient survival rates ${ }^{22}$, as well as modulates oncogenes, tumor suppressor genes, cell cycle regulatory and cell adhesion genes in gastric cancer ${ }^{23}$. Methyl transferase EZH2 promotes methylation of $\mathrm{H} 3 \mathrm{~K} 27 \mathrm{me} 3$ via the PRC2 complex ${ }^{21}$. JARID2, an important component of this complex, plays a pivotal role in its methylation and gene silencing activity ${ }^{21}$. KDM6A, a H3K27me3 demethylase, has been reported to exhibit tumor suppressive role in certain cancers ${ }^{24}$.

The present study reports an increase of H3K27me3 along with EZH2 and JARID2, suggesting an active role of the PRC2 complex. Suppression of KDM6A by chronic exposure to iAs may promote SCC of skin, which may be prevented by BTE. Anomalously, studies in human lung carcinoma cell line A459 showed a repression of H3K27me3 upon iAs exposure ${ }^{25}$. ROS generation also promotes H3K27me3 overexpression ${ }^{26}$. Upregulation of EZH2 was reported in prostrate, breast and head and neck carcinoma ${ }^{27}$, while its inhibition has shown to promote apoptosis via $\mathrm{H} 3 \mathrm{~K} 27 \mathrm{me} 3$ reduction.

$\mathrm{H} 3 \mathrm{~K} 4$ locus has three methylation states, $\mathrm{H} 3 \mathrm{~K} 4 \mathrm{me} 1 / 2 / 3$, which are associated with promoters of the active genes and temporarily stalled genes ${ }^{28}$. In our study, higher expressions of H3K4me1 and MLL3 (the methyltransferase for $\mathrm{H} 3 \mathrm{~K} 4 \mathrm{me} 1$ ) were observed in mice having invasive SCC due to iAs; this may be due to the formation of oncogenic super-enhancers, aggregation of 700 to 1300 bases of H3K4me1 and H3K27ac at the promoter regions of genes ${ }^{28}$. MLL3 promotes mono-methylation of $\mathrm{H} 3 \mathrm{~K} 4$, indirectly inducing oncogenic super-enhancer formation ${ }^{29}$.

Methyl transferase MLL1 plays an important role in methylation of H3K4 locus including its trimethylation ${ }^{30}$. LSD1 demethylates H3K4me1/2 in breast and prostate cancers while its inhibition upregulates tumor suppressor 
activity $^{31}$. Our data reveals downregulation of H3K4me3 and MLL1 in the iAs treated mice. However, in the present study LSD1 did not show any modulation due to BTE. Loss of both H3K4me3 and MLL1 may cause silencing of the tumor suppressor genes and promotion of carcinogenesis ${ }^{28}$. BTE enhances the expression of H3K4me3 and MLL1. This may promote tumor suppressing activity. Abnormally high H3K4me3 (500-3500 bps), an epigenetic signature 'Broad $\mathrm{H} 3 \mathrm{~K} 4 \mathrm{me} 3$ ' is present in the promoters of tumor suppressor genes or cell identity genes, lowering of which has been reported in cancer cells ${ }^{28}$. iAs exposure has shown overexpression of $\mathrm{H} 3 \mathrm{~K} 4 \mathrm{me} 3$ in a lung cancer cell line ${ }^{25}$. It also modulates histone methyl transferases and demethylases altering global expression of $\mathrm{H} 3 \mathrm{~K} 4 \mathrm{me} 3^{32}$.

According to a study, ROS generation led to H3K4me3 overexpression ${ }^{26}$. Repressed H3K4me2/3 correlates with low grade carcinoma and high recurrence of prostate, breast, pancreatic, renal and non-small cell carcinoma $33,34,35,36$. JARID1B, a H3K4me3 demethylase, was found to be upregulated by iAs with a concomitant downregulation of $\mathrm{H} 3 \mathrm{~K} 4 \mathrm{me} 3$, showing its demethylating activity. High expression of JARID1B has been reported in many cancers ${ }^{37}$. Suppression of JARID1B may halt epithelial to mesenchymal transition via upregulation of Ecadherin, thereby preventing carcinogenesis ${ }^{38}$. Our findings show that BTE failed to downregulate the iAs induced elevated level of JARID1B, though prominent upregulation of $\mathrm{H} 3 \mathrm{~K} 4 \mathrm{me} 3$ was observed due to the administration of BTE.

High concentration of JARID1B in cancer stem cells (CSC) expressed oral CSC markers including CD44 and ALDH1 and showed increased PI3K pathway activation ${ }^{39}$. Elevated levels of JARID1B expression were observed in over $87 \%$ of head and neck squamous cell carcinoma cases ${ }^{40}$. We hypothesized that elevation of H3K4me3 by BTE may be due to inhibition of JARID1B, without any impact on its expression. For a better understanding of this phenomenon, in silico studies (docking) with JARID1B and the active molecules in black tea were undertaken. Results show that both theaflavin and theaflavin-3,3'-digallate dock with high affinity with JARID1B. As the $\mathrm{Fe}(\mathrm{II})$ ion is crucial for the demethylation activity of the JmjC domain, theaflavin may have inhibitory effects on the $\mathrm{Fe}$ (II) ion, as it docked very close to the $\mathrm{Mn}$ (II) that replaced $\mathrm{Fe}(\mathrm{II})$ in the crystal structure.

The exact role of the JmjC domain in substrate recognition and binding is still not completely understood, however, our results show that both theaflavin and theaflavin-3,3'-digallate dock in the JmjC domain with high affinity. It has been reported that both of these molecules are transported to the nucleus and interact extensively with histone proteins ${ }^{8}$, hence it is possible that BTE inhibits JARID1B from its demethylation activity due to theaflavin compounds docking at the JmjC domain.

High affinity in docking results does not always prove good protein-ligand docking ${ }^{41}$. Molecular dynamical (MD) simulation is important to analyse the stability of docking, and also to check whether there are significant differences in the docked poses when MD simulations are run for tens of nanoseconds ${ }^{41}$. We did MM/PBSA analysis on Gromacs trajectories to verify the stability of docking. The trajectories of both theaflavin and theaflavin-3,3'-digallate show that the initial poses are maintained after $30 \mathrm{~ns}$ of simulation time. 


\section{Conclusion}

Chronic exposure of iAs in Swiss albino mice resulted in invasive SCC, while no such carcinogenic development was observed in BTE administered mice. iAs, through modulation of certain HMTs, HDMs and HATs, altered repressive and activating histone marks like H3K27me3, H3K4me1, H3K4me3 and H4K16ac respectively. On the other hand, BTE, showed efficacy in modulating these markers, eliciting its chemopreventive role.

JARID1B is an H3K4me2/3 demethylase and has been implicated as a key marker in several cancers, including squamous cell carcinoma ${ }^{40}$. Hence, it is a therapeutic target and it is an active research area to find small molecule ligands that can inhibit the demethylation activity of JARID1B ${ }^{42}$. Though some potential inhibitors have been identified, the pharmacological properties are not well understood ${ }^{42}$. Theaflavin compounds are widely consumed and known to have no side effects, furthermore, these compounds are known to be transported to the nucleus and interact with histone proteins ${ }^{8}$. The in silico studies in this paper raise the possibility that theaflavin compounds may be very effective inhibitors of JARID1B.

\section{Acknowledgement}

The authors are indebted to Director, Chittaranjan National Cancer Institute (CNCI), Kolkata for providing financial support from institutional intramural fund. AG is indebted to CNCI for his fellowship. Authors are also indebted to Director, Chittaranjan National Cancer Institute (CNCI) for infrastructural facilities.

\section{Author contribution}

AG conducted the in vivo experiments and wrote parts of the manuscript, AD did the docking study and parts of the molecular dynamical simulation and wrote parts of the paper, AL conducted parts of the molecular dynamical simulation, analyzed the results, and wrote parts of the paper. SM supervised a part of the in vivo study, MR supervised the in vivo experiments, analyzed the overall results, and wrote parts of the paper.

\section{Competing interests}

Authors confirm that there is no conflict of interest.

\section{Reference}

1. Howe, C. G. \& Gamble, M. V. Influence of Arsenic on Global Levels of Histone Posttranslational Modifications: a Review of the Literature and Challenges in the Field. Curr. Environ. Health Rep. 3(3), $225-37$ (2016).

2. Roy, M., Sinha, D., Mukherjee, S. \& Biswas, J. Curcumin prevents DNA damage and enhances the repair potential in a chronically arsenic-exposed human population in West Bengal, India. Eur. J. Cancer Prev. 20(2), 123-31 (2011).

3. Bhattacharjee, P., Paul, S. \& Bhattacharjee P. Understanding the mechanistic insight of arsenic exposure and decoding the histone cipher. Toxicology. 430, 152340 (2020).

4. Carlos-Reyes, Á. et al. Dietary Compounds as Epigenetic Modulating Agents in Cancer. Front. Genet. 10, 79 (2019)

5. Roy, M. \& Datta, A. Cancer Genetics and Therapeutics - focus on phytochemicals. (Springer 2019).

6. Sinha, D., Roy, S. \& Roy, M. Antioxidant potential of tea reduces arsenite induced oxidative stress in Swiss albino mice. Food Chem. Toxicol. 48(4), 1032-9 (2010).

7. Wu, Y. Y., Li, W., Xu, Y., Jin, E. H. \& Tu, Y. Y. Evaluation of the antioxidant effects of four main theaflavin derivatives through chemiluminescence and DNA damage analyses. J. Zhejiang Univ. Sci. B. 12(9), 744-51 (2011).

8. Mikutis, G. et al. Phenolic promiscuity in the cell nucleus--epigallocatechingallate (EGCG) and theaflavin-3,3'digallate from green and black tea bind to model cell nuclear structures including histone proteins, double stranded DNA and telomeric quadruplex DNA. Food Funct. 4(2), 328-37 (2011). 
9. Allred, D. C., Harvey, J. M., Berardo, M. \& Clark, G. M. Prognostic and predictive factors in breast cancer by immunohistochemical analysis. Mod. Pathol. 11, 155-68 (1998).

10. Johansson, C. et al. Structural analysis of human KDM5B guides histone demethylase inhibitor development. Nat. Chem. Biol. 12(7), 539-45 (2016).

11. Sanner, M. F. Python: A programming language for software integration and development. J. Mol. Graphics Mod. 17, 57-61 (1999).

12. Kochnev, Y., Hellemann, E., Cassidy, K. C. \& Durrant, J. D. Webina: an open-source library and web app that runs AutoDock Vina entirely in the web browser. Bioinformatics. 36(16), 4513-15 (2020).

13. Trott, O. \& Olson, A. J. AutoDock Vina: improving the speed and accuracy of docking with a new scoring function, efficient optimization and multithreading. J. Comput. Chem. 31, 455-61 (2010).

14. Abraham, M. J. et al. GROMACS: High performance molecular simulations through multi-level parallelism from laptops to supercomputers. SoftwareX. 1-2, 19-25 (2015).

15. Kumari, R., Kumar, R. \& Lynn, A. g_mmpbsa- A GROMACS tool for high-throughput MM-PBSA calculations. $J$. Chem. Inf. Model. 54(7), 1951-62 (2014).

16. Khan, S. A., Reddy, D. \& Gupta, S. Global histone post-translational modifications and cancer: Biomarkers for diagnosis, prognosis and treatment? World J. Biol. Chem. 6(4), 333-45 (2015).

17. Miller, K. M. \& Jackson, S. P. Histone marks: repairing DNA breaks within the context of chromatin. Biochem. Soc. Trans. 40, 370-6 (2012).

18. Wu, D., Qiu, Y., Jiao, Y., Qiu, Z. \& Liu, D. Small Molecules Targeting HATs, HDACs, and BRDs in Cancer Therapy. Front Oncol. 10, 560487 (2020).

19. Liu, N. et al. A potential diagnostic marker for ovarian cancer: Involvement of the histone acetyltransferase, human males absent on the first. Oncol. Lett. 6(2), 393-400 (2013).

20. Ghosh, A., Mukherjee, S., Roy, M. \& Datta, A. Modulatory role of tea in arsenic induced epigenetic alterations in carcinogenesis. Nucleus. 64, 143-56 (2021).

21. Sanulli, S. et al. JARID2 Methylation via the PRC2 Complex Regulates H3K27me3 Deposition during Cell Differentiation. Mol. Cell. 57(5), 769-83 (2015).

22. Tzao, C. et al. Prognostic significance of global histone modifications in resected squamous cell carcinoma of the esophagus. Mod. Pathol. 22, 252-60 (2009).

23. Zhang, L., Zhong, K., Dai, Y. \& Zhou, H. Genome-wide analysis of histone H3 lysine 27 trimethylation by ChIPchip in gastric cancer patients. J. Gastroenterol. 44, 305-12 (2009).

24. Andricovich, J. et al. Loss of KDM6A Activates Super-Enhancers to Induce Gender-Specific Squamous-like Pancreatic Cancer and Confers Sensitivity to BET Inhibitors. Cancer Cell. 33(3), 512-26 (2018).

25. Zhou, X., Sun, H., Ellen, T. P., Chen, H. \& Costa, M. Arsenite alters global histone H3 methylation. Carcinogenesis. 29(9), 1831-36 (2008).

26. Niu, Y., Desmarais, T. L., Tong, Z., Yao, Y. \& Costa, M. Oxidative stress alters global histone modification and DNA methylation. Free Radic. Biol. Med. 82, 22-8 (2015).

27. Mochizuki, D. et al. Aberrant Epigenetic Regulation in Head and Neck Cancer Due to Distinct EZH2 Overexpression and DNA Hypermethylation. Int J Mol Sci. 19(12), 3707 (2018).

28. Dhar, S. S. et al. MLL4 Is Required to Maintain Broad H3K4me3 Peaks and Super-Enhancers at Tumor Suppressor Genes. Mol. Cell. 70(5), 825-41 (2018).

29. Jia, Q., Chen, S., Tan, Y., Li, Y. \& Tang, F. Oncogenic super-enhancer formation in tumorigenesis and its molecular mechanisms. Exp. Mol. Med. 52(5), 713-23 (2020).

30. Fang, Y. et al. KMT2A histone methyltransferase contributes to colorectal cancer development by promoting cathepsin Z transcriptional activation. Cancer Med. 8(7), 3544-52 (2019).

31. Lim, S. et al. Lysine-specific demethylase 1 (LSD1) is highly expressed in ER-negative breast cancers and a biomarker predicting aggressive biology. Carcinogenesis. 31, 512-20 (2010).

32. Tu, W., Liu, Y., Xie, C. \& Zhou, X. Arsenite downregulates H3K4 trimethylation and H3K9 dimethylation during transformation of human bronchial epithelial cells. J. Appl. Toxicol. 38, 480-8 (2018).

33. Barlési, F. et al. Global histone modifications predict prognosis of resected non small-cell lung cancer. J Clin. Oncol. 25, 4358-64 (2007).

34. Elsheikh, S. E. et al. Global histone modifications in breast cancer correlate with tumor phenotypes, prognostic factors, and patient outcome. Cancer. Res. 69, 3802-09 (2009).

35. Ellinger, J. et al. Prognostic relevance of global histone H3 lysine 4 (H3K4) methylation in renal cell carcinoma. Int. J. Cancer. 127, 2360-66 (2010).

36. Manuyakorn, A. et al. Cellular histone modification patterns predict prognosis and treatment response in resectable pancreatic adenocarcinoma: results from RTOG 9704. J. Clin. Oncol. 28, 1358-65 (2010).

37. Zhang, J. Overexpression of JARID1B promotes differentiation via SHIP1/AKT signaling in human hypopharyngeal squamous cell carcinoma. Cell Death Dis. 7(9), e2358 (2016).

38. Tang, B. JARID1B promotes metastasis and epithelial-mesenchymal transition via PTEN/AKT signaling in hepatocellular carcinoma cells. Oncotarget. 6(14), 12723-39 (2015).

39. Facompre, N. D. et al. JARID1B Enables Transit between Distinct States of the Stem-like Cell Population in Oral Cancers. Cancer Res. 76(18), 5538-49 (2016). 
40. Cui, Z. et al. PLU-1/ JARID1B overexpression predicts proliferation properties in head and neck squamous cell carcinoma. Oncol. Rep. 33, 2454-60 (2015).

41. Chen, Y. C. Beware of docking! Trends Pharmacol. Sci. 36(2), 78-95 (2015).

42. Sayegh, J. et al. Identification of Small Molecule Inhibitors of Jumonji AT-rich Interactive Domain 1B (JARID1B) Histone Demethylase by a Sensitive High Throughput Screen. The J Biological Chemistry. 288(13), 9408-9417 (2013). 


\section{Supplementary Files}

This is a list of supplementary files associated with this preprint. Click to download.

- WBrawdata.pdf

- theaflavin.mov

- theaflavin33digallate.mov 